\title{
Localized exciton and its stimulated emission in surface mode from single-layer $\operatorname{In}_{x} \operatorname{Ga}_{1-x} \mathrm{~N}$
}

\author{
Akihiro Satake and Yasuaki Masumoto \\ Institute of Physics, University of Tsukuba, Tsukuba, Ibaraki 305, Japan
}

\author{
Takao Miyajima, Tsunenori Asatsuma, Fumihiko Nakamura, and Masao Ikeda \\ Research Center, Sony Corporation, Yokohama, Kanagawa 240, Japan \\ (Received 11 June 1997; revised manuscript received 4 August 1997)
}

\begin{abstract}
Exciton localization in $\operatorname{In}_{x} \mathrm{Ga}_{1-x} \mathrm{~N}$ was studied. At $2 \mathrm{~K}$, the time-integrated photoluminescence (PL) spectrum showed a Stokes shift from the absorption shoulder and broadening at the lower photon energy side. Site-selectively excited PL measurements determined the mobility edge. The exciton relaxation processes were studied by use of time-resolved PL spectroscopy. The PL decay time increased with the decrease of the detection-photon energy, indicating the dynamical features of exciton localization. In addition, we observed localized exciton luminescence turned into stimulated emission just below the mobility edge. [S0163-1829(98)51004-1]
\end{abstract}

\section{INTRODUCTION}

Recently, III-V nitride compound semiconductors have been recognized as very important materials for fabricating short-wavelength-emitting devices such as light-emitting diodes (LED's) and laser diodes (LD's) in the ultraviolet/blue region. $\mathrm{In}_{x} \mathrm{Ga}_{1-x} \mathrm{~N}$ is a promising material for light-emitting devices because its direct band gap energy varies from 1.9 $\mathrm{eV}$ (band gap of InN) to $3.4 \mathrm{eV}$ (band gap of $\mathrm{GaN}$ ) with the change of the alloy composition. ${ }^{1} \operatorname{In}_{x} \mathrm{Ga}_{1-x} \mathrm{~N}$ single quantum wells and multiple quantum wells have already been used as the active layer for blue/green LED's and blue LD's. ${ }^{2-4}$ Localized excitons have been proposed to give a major spontaneous emission in $\operatorname{In}_{x} \mathrm{Ga}_{1-x} \mathrm{~N} .^{5-8}$ However, fundamental optical properties of $\operatorname{In}_{x} \mathrm{Ga}_{1-x} \mathrm{~N}$ have not been studied substantially. Therefore it is necessary to study the properties of the optical transition and their dynamics in $\operatorname{In}_{x} \mathrm{Ga}_{1-x} \mathrm{~N}$ ternary alloys. This study provides better understanding of not only fundamental optical properties, but also lasing mechanisms.

In ternary alloys, the effect of disorder on electronic states of materials can be observed in the band edge region through the broadening of optical spectra due to formation of the band tail states. ${ }^{9-12}$ The band tail states lead to exciton localization, which strongly influences recombination and transport properties of materials. Furthermore, the localized excitons can contribute to the optical gain, because the lowdensity tail states are easily occupied by the moderate pumping. ${ }^{13-15}$ In this case the relative energy position between the mobility edge and the optical gain is an important parameter for judging whether the excitons or the localized excitons contribute to the optical gain.

In this paper, we investigated photoluminescence (PL) of single-layer $\mathrm{In}_{x} \mathrm{Ga}_{1-x} \mathrm{~N}$ grown by metal-organic chemical vapor deposition (MOCVD), and discussed the exciton relaxation processes. In addition, we determined the exciton mobility edge and observed stimulated emission just below the mobility edge in surface emitting configuration.

\section{EXPERIMENTAL PROCEDURE}

The samples used in this study were grown by MOCVD on sapphire substrates with GaN buffer layers. Trimethylgallium (TMGa), trimethylindium (TMIn) and ammonia $\left(\mathrm{NH}_{3}\right)$ were used as $\mathrm{Ga}$, In, and $\mathrm{N}$ sources, respectively. Sample 1 consists of an undoped single-layer $\mathrm{In}_{0.08} \mathrm{Ga}_{0.92} \mathrm{~N} 360 \mathrm{~nm}$ thick on a GaN layer $1 \mu \mathrm{m}$ thick, and is covered by a GaN cap layer $50 \mathrm{~nm}$ thick. Sample 2 consists of an undoped single-layer $\operatorname{In}_{0.14} \mathrm{Ga}_{0.86} \mathrm{~N} 200 \mathrm{~nm}$ thick on a $\mathrm{GaN}$ layer 1.5 $\mu \mathrm{m}$ thick. The $\operatorname{In}_{x} \mathrm{Ga}_{1-x} \mathrm{~N}$ layer was deposited at temperature ranging from 700 to $800{ }^{\circ} \mathrm{C}$. The samples were directly immersed in superfluid helium at $2 \mathrm{~K}$.

Site-selectively excited PL measurement and timeresolved PL spectroscopy were performed by use of secondharmonic pulses of a mode-locked Ti:sapphire laser pumped by a cw $\mathrm{Ar}^{+}$laser. The pulse duration and repetition rate were about 2 ps and $82 \mathrm{MHz}$, respectively. The photon energy of the laser output can be tuned from 1.5 to $1.7 \mathrm{eV}$ to give second-harmonic pulses ranging from 3.0 to $3.4 \mathrm{eV}$. For the time-resolved PL spectroscopy, a synchroscan streak camera in conjunction with a subtractive dispersion double monochromator was used. The time resolution was about $\sim 30 \mathrm{ps}$. In order to make assignment of emissions under the high-density excitation, second-harmonic pulses of the Ti:sapphire regenerative amplifier output were used as the excitation source. The pulse duration and repetition rate were $300 \mathrm{fs}$ and $200 \mathrm{kHz}$, respectively.

\section{RESULTS AND DISCUSSION}

In ternary alloys, the spatial potential fluctuations due to composition fluctuations can localize excitons. The density of localized states is considered to be approximated by an exponential tail. ${ }^{9}$ In order to determine the mobility edge, we took PL spectra by changing the excitation photon energy. Figure 1 shows the PL spectra of sample 1 measured at different excitation photon energies $(3.122 \sim 3.305 \mathrm{eV})$. The PL peak position depends on the excitation photon energy, as is shown in the inset of Fig. 1. Above $3.175 \mathrm{eV}$, the PL peak 


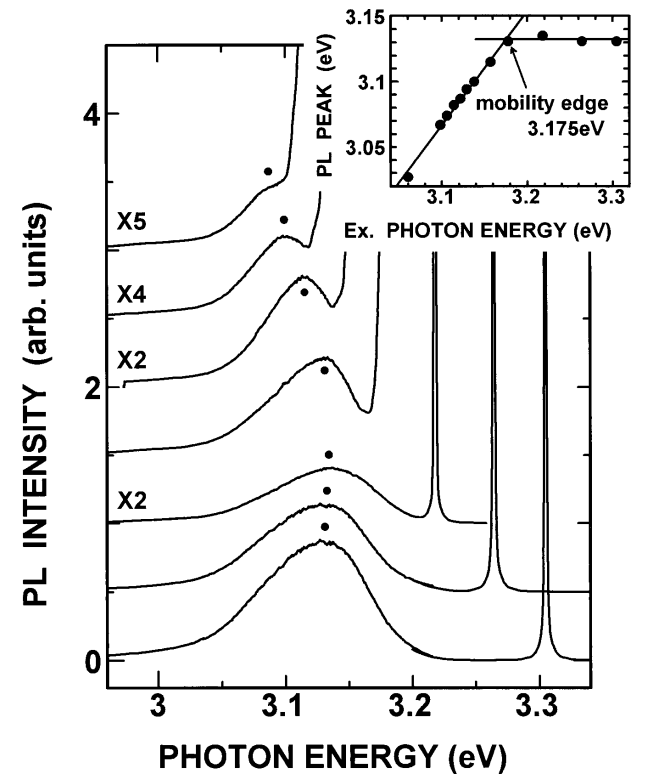

FIG. 1. PL spectra of sample $1(x=0.08)$ under the site-selective excitation $(3.122 \sim 3.305 \mathrm{eV})$. Closed circles show the PL peaks. The inset shows the PL peak energy as a function of the excitation photon energy.

does not move with the change of the excitation photon energy. Then, the PL peak is located at $3.13 \mathrm{eV}$ and a full width at half-maximum (FWHM) is about $95 \mathrm{meV}$. The PL spectra are broadened toward the lower photon energy side. Below $3.175 \mathrm{eV}$, the PL peak shows a redshift with the decrease of the excitation photon energy. We can determine the mobility edge from the results of Fig. 1 and it is located at $3.175 \mathrm{eV}$.

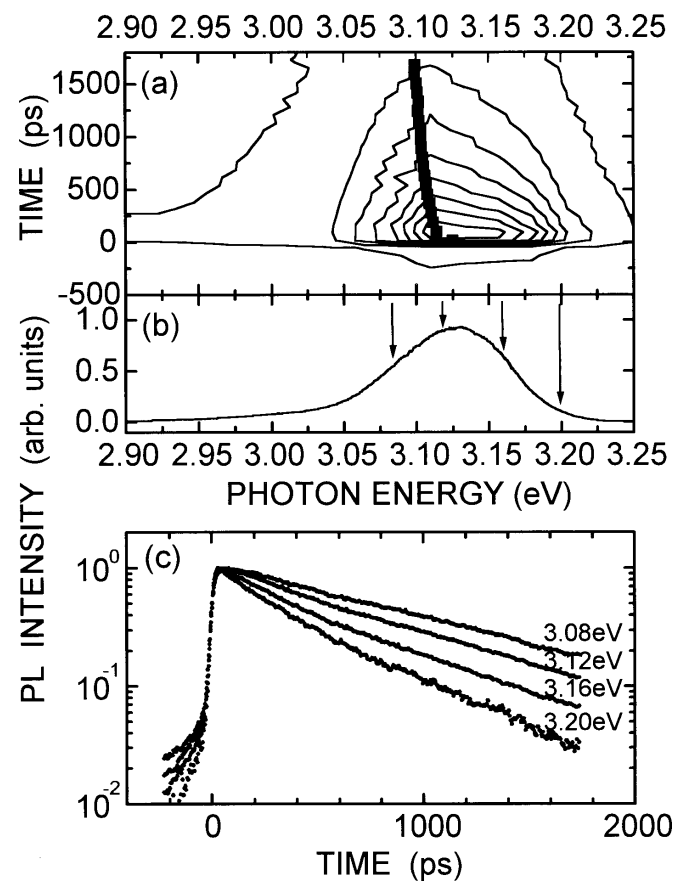

FIG. 2. (a) A contour map of the energy- and time-resolved PL intensity of sample $1(x=0.08)$. Closed squares show the PL center of gravity as a function of time. (b) Time-integrated PL spectrum. (c) Temporal change of the PL observed at 3.08, 3.12, 3.16, and $3.20 \mathrm{eV}$ [indicated by arrows in (b)].
The energy- and time-resolved PL of sample 1 was shown in Fig. 2(a). The excitation photon energy is $3.382 \mathrm{eV}$, which is lower than the band gap of the GaN layer. In this condition, we can discuss the carrier dynamics only in the $\mathrm{In}_{x} \mathrm{Ga}_{1-x} \mathrm{~N}$ layer. The closed squares in Fig. 2(a) indicate the PL center of gravity as a function of time. It shifts toward lower photon energy as time proceeds. After 200 ps, the decrease rate of photon energy slows down and is $1 \times 10^{7}$ $\mathrm{eV} / \mathrm{s}$. The energy loss goes on during the carrier lifetime. The PL decay can be described nearly by a singleexponential decay function as shown in Fig. 2(c). The decay time of PL increases with the decrease of the detection photon energy.

The experimental results are interpreted by an energy relaxation model for localized excitons. The relaxation processes of localized excitons are explained as follows. After the intraband excitation, excitons are formed in the extended states and have a kinetic energy far exceeding the amplitude of potential fluctuations. Such excitons can be regarded as free composite particles and have a high probability of spatial migration. The excitons relax quickly into the localized states where further relaxation and spatial migration are possible only through the tunneling between the localized states. The relaxation rate for such a process strongly depends on the density of available final states with lower energy and decreases quickly with the decrease of energy within the tail due to the low density of the final states. The decay rate of localized excitons is expressed by radiative recombination decay rate plus relaxation rate to the lower-energy states. Therefore, the decay time increases with the decrease of the detection photon energy and the center of gravity of PL shifts toward lower energy as time proceeds.

When In concentration $x$ increases, the band gap is expected to become smaller and the spectral broadening is expected to become larger. Figure 3 shows the energy dependence of the PL decay time in both of samples [Fig. 3(a) for sample 1 and (b) for sample 2] under the low-density excitation (picosecond-pulse excitation). The absorption (dashed lines) and the PL spectra (solid lines) were modulated by Fabry-Perot interference effects of thin films, but we got rid of the Fabry-Perot modes and restored the intrinsic spectra by simple calculation. The mobility edge $E_{\text {me }}$ of sample 2 $\left(x=0.14: E_{\mathrm{me}}=3.07 \mathrm{eV}\right)$ is lower than that of sample 1 $\left(x=0.08: E_{\mathrm{me}}=3.175 \mathrm{eV}\right)$. The absorption shoulder and the PL peak of sample 2 are also located at the lower photon energy side than those of sample 1. The PL spectrum of sample 2 is broader than that of sample 1 (full widths at half-maximum are $95 \mathrm{meV}$ for sample 1 and $200 \mathrm{meV}$ for sample 2). Below the mobility edge, the absorption spectra for both samples are considered to be approximated by

$$
D(E)=A \exp \left(\frac{E-E_{\mathrm{me}}}{\epsilon}\right),
$$

where $D(E)$ is the optical density spectrum, and $\epsilon$ is the characteristic energy indicating the broadening of the tail state. The absorption tails are well fitted by the expression as shown in Fig. 3. The fitting parameters are $\epsilon=21 \mathrm{meV}$ and $A=0.31$ for sample 1 and $\epsilon=93 \mathrm{meV}$ and $A=0.51$ for sample 2. Thus, these optical properties of $\operatorname{In}_{x} \mathrm{Ga}_{1-x} \mathrm{~N}$ alloys 

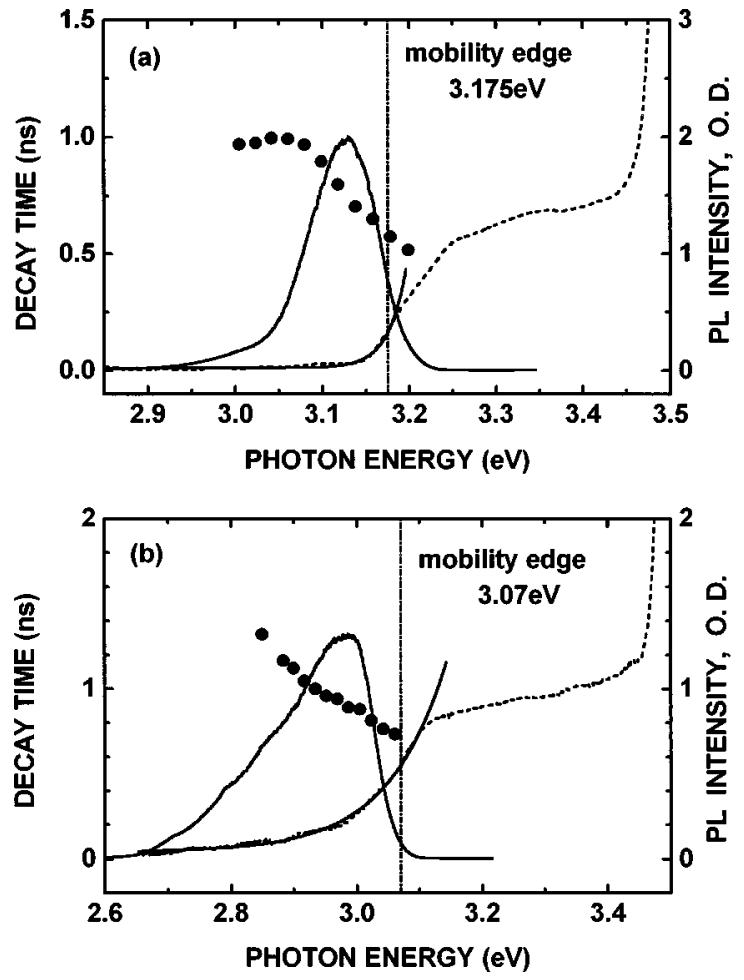

FIG. 3. (a) Absorption (a dashed line) and PL (a solid line) spectra of sample $1(x=0.08)$. They are gotten rid of by the FabryPerot modes and are restored by calculation. Closed circles show the decay time observed at various photon energies. The absorption tail is fitted by Eq. (1) in the text. (b) Same as (a) for sample 2 $(x=0.14)$

are consistent with the compositional disorder model. The exciton localization is caused by the compositional fluctuation for both samples.

As the density of localized states in the band tail is lower than that of the extended band states, it can be easily filled by optical pumping. If the optical gain coming from the filled localized states exceeds the light propagation loss, stimulated emission sets in. This phenomenon has been observed in

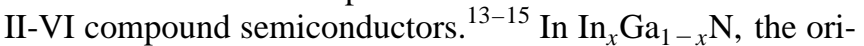
gin of stimulated emission is a very interesting issue, but it has not been clarified yet. Although localized excitons have recently been proposed contributing to the lasing mechanism for $\mathrm{In}_{x} \mathrm{Ga}_{1-x} \mathrm{~N}$ LD's, ${ }^{6,8}$ there is so far no direct experimental support for this claim. The ambiguity mainly comes from the lack of the experimental determination of the mobility edge. In the above procedures, we successfully determined the mobility edge. Further, we have studied the stimulated emission from the $\operatorname{In}_{x} \mathrm{Ga}_{1-x} \mathrm{~N}$ layer. Under the high-density excitation, we could observe stimulated emission in the surface emitting configuration. Figure 4 shows the excitation intensity dependence of time-integrated PL spectra of sample 2. The excitation photon energy is $3.323 \mathrm{eV}$. When the excitation energy density is $2.5 \mu \mathrm{J} / \mathrm{cm}^{2}$, the PL spectrum has a broad emission band. The peak is located at $3.01 \mathrm{eV}$ and FWHM is about $200 \mathrm{meV}$. The PL decay is nearly a single-exponential decay as shown in Fig. 5(a). The decay time of PL increases with the decrease of the detection photon energy, which is similar to the feature of decay time under the picosecondpulse excitation. Increasing the excitation energy density, the

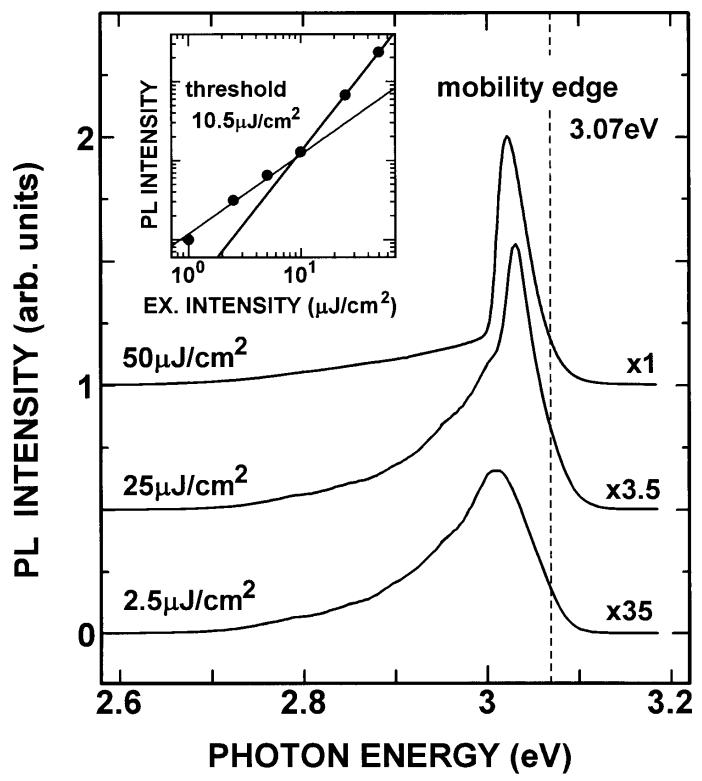

FIG. 4. PL spectra of sample 2 for different excitation energy densities. The Fabry-Perot modes are eliminated by calculation. The inset shows the excitation intensity dependence of the PL intensity. The stimulated emission appears at $I_{\mathrm{th}}=10.5 \mu \mathrm{J} / \mathrm{cm}^{2}$.

PL intensity increases linearly below the threshold $\left(\mathrm{I}_{\mathrm{th}}=10.5\right.$ $\mu \mathrm{J} / \mathrm{cm}^{2}$ ). On the other hand, above the threshold, a superlinearly grown sharp emission band appeared. It is located at $3.03 \mathrm{eV}$, which is just below the mobility edge and its FWHM is about $30 \mathrm{meV}$ under the excitation energy density of $\mathrm{I}_{\mathrm{ex}}=25 \mu \mathrm{J} / \mathrm{cm}^{2}$. The decay time of the emission is less than $\sim 30 \mathrm{ps}$, the time resolution of the experiment, as shown in Fig. 5(b). The temporal trace indicates a change from spontaneous emission to stimulated one. The stimulated emission shows a slight redshift with the increase of the excitation energy density.

We consider that the stimulated emission from the $\mathrm{In}_{x} \mathrm{Ga}_{1-x} \mathrm{~N}$ layer comes from the localized excitons because the stimulated emission is observed just below the mobility edge. The reason why the optical gain is largest just below the mobility edge can be explained as follows. The photogenerated excitons lose their energy quickly, when the exciton energy is higher than the mobility edge. This is because the excitons can find easily the lower-energy states by their mi-

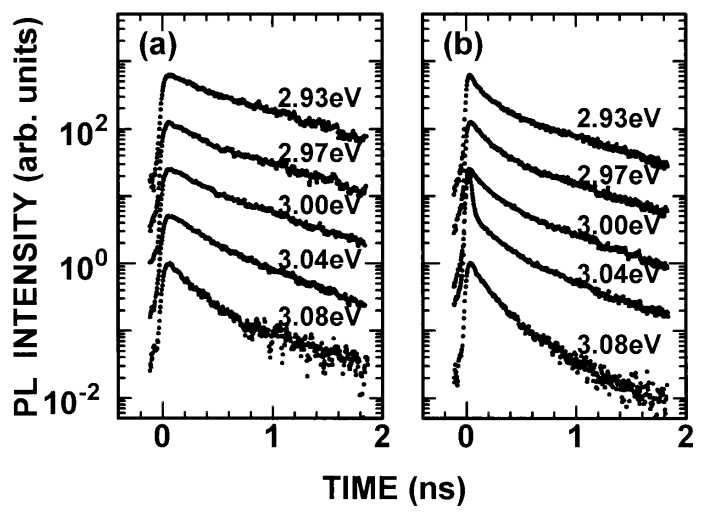

FIG. 5. (a) Time-resolved PL under the low-density excitation $I_{\mathrm{ex}}=2.5 \mu \mathrm{J} / \mathrm{cm}^{2}$. (b) Time-resolved PL under the high-density excitation $I_{\mathrm{ex}}=25 \mu \mathrm{J} / \mathrm{cm}^{2}$. 
gration. On the other hand, the exciton energy relaxation becomes slow, because the energy relaxation mode turns to tunneling between the low-density localized exciton states. The potential depth and barrier depend on the concentration of In, which changes with the change of the spatial position. The photogenerated excitons are localized at the positions where the exciton energy decreases and passes through the mobility edge. As a result, localized excitons occupy every localized tail state equally. As the first approximation, the energy distribution function of excitons is represented neither by Fermi distribution function nor by the Boltzmann function, but by a constant. Then, the optical gain has the maximum value just below the mobility edge and has the tail in the lower-energy side. The asymmetric optical gain spectrum increases with the increase of the excitation energy density. The optical gain decays fast at the higher-energy side, but slow at the lower-energy side. This is because the rather slow energy relaxation between the localized exciton states takes place. Therefore, the total gain shows up just like a redshift with the increase of the excitation energy density.
Although the above mentioned scenario is rather crude, the observed features are well understood by the scenario.

\section{CONCLUSION}

We studied the exciton localization in single-layer $\mathrm{In}_{x} \mathrm{Ga}_{1-x} \mathrm{~N}$ grown by MOCVD, by use of the site-selectively excited PL measurement and the time-resolved PL spectroscopy. The mobility edges of samples were determined at $3.175 \mathrm{eV}$ for sample $1(x=0.08)$ and $3.07 \mathrm{eV}$ for sample 2 $(x=0.14)$, respectively. The feature of the decay time under low-density excitation was interpreted by the exciton localization to the band tail states. Under the high-density excitation, we observed the surface mode stimulated emission just below the mobility edge. The decay time was less than $\sim 30$ ps. The stimulated emission is attributed to the localized excitons. However, the optical gain mechanism is not still clear enough because of the lack of the optical gain spectra data. In order to understand the optical gain mechanism, more detailed experimental and theoretical analyses are required.
${ }^{1}$ K. Osamura, K. Nakajima, Y. Murakami, P. H. Shingu, and A. Ohtsuki, Solid State Commun. 11, 617 (1972).

${ }^{2}$ S. Nakamura, M. Senoh, N. Iwasa, S. Nagahama, T. Yamada, and T. Mukai, Jpn. J. Appl. Phys., Part 34, L1332 (1995).

${ }^{3}$ S. Nakamura, M. Senoh, S. Nagahama, N. Iwasa, T. Yamada, T. Matsushita, H. Kiyoku, and Y. Sugimoto, Jpn. J. Appl. Phys., Part 35, L74 (1996).

${ }^{4}$ S. Nakamura, M. Senoh, S. Nagahama, N. Iwasa, T. Yamada, T. Matsushita, Y. Sugimoto, and H. Kiyoku, Appl. Phys. Lett. 70, 1417 (1997).

${ }^{5}$ M. Smith, G. D. Chen, J. Y. Lin, H. X. Jiang, M. Asif Khan, and Q. Chen, Appl. Phys. Lett. 69, 2837 (1996).

${ }^{6}$ S. Chichibu, T. Azuhata, T. Sota, and S. Nakamura, Appl. Phys. Lett. 69, 4188 (1996)

${ }^{7}$ Y. Narukawa, Y. Kawakami, Sz. Fujita, Sg. Fujita, and S. Naka- mura, Phys. Rev. B 55, R1938 (1997).

${ }^{8}$ Y. Narukawa, Y. Kawakami, M. Funato, Sz. Fujita, Sg. Fujita, and S. Nakamura, Appl. Phys. Lett. 70, 981 (1997).

${ }^{9}$ S. Permogorov and A. Reznitsky, J. Lumin. 52, 201 (1992).

${ }^{10}$ E. Cohen and M. D. Sturge, Phys. Rev. B 25, 3828 (1982).

${ }^{11}$ J. A. Kash, A. Ron, and E. Cohen, Phys. Rev. B 28, 6147 (1983).

${ }^{12}$ S. Yamaguchi, Y. Kawakami, Sz. Fujita, Sg. Fujita, Y. Yamada, T. Mishina, and Y. Masumoto, Phys. Rev. B 54, 2629 (1996).

${ }^{13}$ F. A. Majumder, S. Shevel, V. G. Lyssenko, H. E. Swoboda, and C. Klingshirn, Z. Phys. B 66, 409 (1987).

${ }^{14}$ J. Ding, H. Jeon, T. Ishihara, M. Hagerott, A. V. Nurmikko, H. Luo, N. Samarth, and J. Furdyna, Phys. Rev. Lett. 69, 1707 (1992).

${ }^{15}$ Y. Yamada, Y. Masumoto, J. T. Mullins, and T. Taguchi, Appl. Phys. Lett. 61, 2190 (1992). 